Canadian University Music Review

Revue de musique des universités canadiennes

\title{
Une grille d'analyse des musiques électro-acoustiques
}

\section{François Guérin}

Volume 10, numéro 1, 1990

URI : https://id.erudit.org/iderudit/1014896ar

DOI : https://doi.org/10.7202/1014896ar

Aller au sommaire du numéro

\section{Éditeur(s)}

Canadian University Music Society / Société de musique des universités

canadiennes

\section{ISSN}

0710-0353 (imprimé)

2291-2436 (numérique)

Découvrir la revue

\section{Citer cet article}

Guérin, F. (1990). Une grille d'analyse des musiques électro-acoustiques. Canadian University Music Review / Revue de musique des universités

canadiennes, 10(1), 71-100. https://doi.org/10.7202/1014896ar

All Rights Reserved (C Canadian University Music Society / Société de musique des universités canadiennes, 1990
Ce document est protégé par la loi sur le droit d'auteur. L’utilisation des services d'Érudit (y compris la reproduction) est assujettie à sa politique d'utilisation que vous pouvez consulter en ligne.

https://apropos.erudit.org/fr/usagers/politique-dutilisation/ 


\title{
UNE GRILLE D'ANALYSE DES MUSIQUES ÉLECTROACOUSTIQUES
}

\author{
François Guérin
}

\section{INTRODUCTION}

On sait combien l'introduction des musiques électroacoustiques a profondément modifié les habitudes perceptives liées à la musique. Tous les paramètres sonores pouvant désormais être exploités à des fins musicales, tous les caractères sonores pouvant être explorés pour eux-mêmes, les critères perceptifs, qui avaient jusque-là guidé l'écoute de la musique, ne pouvaient suffire pour ces nouvelles constructions musicales.

Cependant, un découpage perceptif s'opère tout de même à l'écoute des musiques électroacoustiques. L'auditeur parvient à segmenter l'information, à assimiler des données sonores nouvelles, bref à saisir jusqu'à un certain point ce qu'on lui donne à entendre, quelle que soit par ailleurs la nouveauté ou la difficulté de la musique.

Quels sont alors les mécanismes perceptifs auxquels recourt l'auditeur, pour affronter ces musiques? À l'aide de quels critères parvient-il à effectuer une reconnaissance des formes ou des structures musicales? Sur quoi s'appuie finalement sa compréhension, même partielle, des musiques électroacoustiques?

Ces questions sont à considérer dans un cadre plus général. La difficulté à analyser les musiques contemporaines, malgré quelques brillantes applications ça et là, est bien perçue par les musicologues, et a donné lieu à quelques propositions.

On connaît la classification morpho-typologique de Pierre Schaeffer (1966), sans doute une des tentatives les plus achevées pour décrire les sons. Mais la description n'est qu'une étape de l'analyse. Lerdhal et Jackendoff (1983) admettent que leur ouvrage, sur la théorie générative de la musique tonale, concerne en premier lieu celle-ci, même s'ils souhaitent que les principes énoncés puissent s'appliquer à un plus large éventail de langages musicaux. David Wessel (1979) cherche à classer les timbres dans une représentation spatiale à trois dimensions afin d'établir les relations entre eux. Deutsch 
(1982), Bregman (1978), Cooper (1960), Plomp (1976), Cogan et Escott (1976) et nombre d'autres, tentent de démontrer le rôle des variables individuelles sur la perception.

Mais après les diverses tentatives de classification basées sur les concepts de la linguistique ou encore, sur les théories de Schenker, toutes dépendantes de la partition, on doit surtout retenir les approches qui reposent sur ce que l'oreille perçoit, d'abord et avant tout. Longtemps rejeté à cause de la composante subjective qu'il induit, cet aspect, comme en avait déjà discuté Schaeffer, est sans doute à considérer maintenant comme primordial pour trancher dans les directions d'analyse qui s'offrent aux chercheurs. Arom (1985), Erickson (1975), Thoresen (1985), Delalande (1976) font partie des auteurs qui insistent sur la nécessité de garder les objectifs de l'analyse à l'intérieur des limites perceptives de l'oreille.

Cette orientation offre deux avantages. Elle permet d'accepter dans le champ d'études toutes les musiques qui ne possèdent pas le support de la partition, ou encore, celles dont la représentation graphique est très éloignée des conventions d'écriture reconnues par les musicologues, puisque l'analyse peut reposer sur ce que l'oreille entend, peu importe ce qui est donné à entendre. Elle permet ensuite d'ouvrir une perspective de recherche prometteuse : si l'oreille devient ainsi le pivot du travail d'analyse, on peut se demander comment celle-ci fonctionne, comment elle appréhende l'information sonore qui lui parvient, selon quels critères elle retient, ne retient pas ou met en relation, les traits perceptifs qu'elle identifie au fur et à mesure du déroulement musical.

Dans cet article, nous tenterons d'établir quels sont les éléments constitutifs d'une structure musicale électroacoustique qui sont pertinents à l'écoute, qui sont retenus dans la perception, qui permettent de focaliser l'intérêt de l'auditeur. Cette recherche est basée sur une expérience au cours de laquelle des sujets ont été confrontés à des extraits de musique électroacoustique, afin de définir quels sont les traits perceptifs caractéristiques qui se dégagent lors d'un acte de perception appliqué à ces musiques.

\section{PRÉSENTATION DE L'EXPÉRIENCE}

L'angle d'approche de cette expérience consiste à demander à des sujets d'expliquer ce qu'ils apprécient ou non dans divers extraits de musique électroacoustique. En procédant ainsi, un sujet est obligé de cerner et d'expliciter 
ce qu'il retient de son écoute et de définir les critères qu'il utilise pour établir son appréciation et pour porter un jugement.

L'objectif de l'expérience est de parvenir à recenser le plus grand nombre possible des critères qui interviennent dans la perception de ces musiques, et de tenter d'établir autour de quels axes ces critères se regroupent. Par là, il nous sera possible d'extrapoler les résultats et de proposer certaines hypothèses.

Le terme critère est l'élément-clé de notre recherche. Un critère est basé sur différents indices identifiés dans un déroulement musical. Ces indices permettent à l'auditeur d'opérer un découpage, une identification d'éléments pertinents, afin qu'il puisse traiter adéquatement l'information musicale qu'on lui transmet. Tout acte perceptif fait donc intervenir une série de critères qui concernent chacun des aspects différents de la structure musicale, selon les indices et les intentions liés à l'écoute. Tous les critères impliqués fonctionnent de pair et concourent à la réussite de l'acte perceptif.

Dans un premier temps, l'expérience visera à recueillir le plus de réponses descriptives possibles. Dans un deuxième temps, ces réponses seront regroupées en critères distincts. Nous aurons alors un profil élaboré de ce sur quoi repose la perception des musiques électroacoustiques.

\section{A) Les sujets}

Pour les besoins de cette expérience, 11 sujets familiers avec le domaine de la musique électroacoustique ont été mis à contribution. La plupart d'entre eux étaient même déjà compositeurs ou en voie de formation. Leur connaissance du genre était suffisamment développée pour qu'ils puissent dépasser le premier niveau d'écoute et apporter à leurs réponses une dimension réfléchie.

Il est important de préciser que cette expérience avait déjà été conduite avec des sujets choisis au hasard. Cette façon de faire, si elle respecte les conditions expérimentales de base, s'est avérée plus ou moins profitable compte tenu de leur expérience limitée, pour la plupart, du domaine électroacoustique. Devant la disparité et l'imprécision des réponses, nous avons décidé d'adopter le parti inverse et de choisir cette fois des sujets versés dans les musiques électroacoustiques, des sujets donc habitués à percevoir ces musiques avec un jugement critique et un vocabulaire mieux adapté. Contrairement à des sujets pris au hasard, à qui on demandait une tâche qui requérait une certaine expertise, et qui s'en acquittaient du mieux qu'ils le pouvaient au risque de choisir des termes impropres, des sujets sélectionnés en fonction d'une certaine compétence musicale étaient en mesure de proposer des réponses plus concises, plus directes et mieux ordonnées, puisqu'elles reposaient 
Figure 1

Liste des extraits musicaux de l'expérience

1. Jean SCHWARZ: ERDA: 54 oiseaux

$48 \mathrm{~s}$

2. Pierre HENRY: LA NOIRE À 60

$30 \mathrm{~s}$

3. Jacques LEJEUNE: PARAGES: Traces et réminiscences

$34 \mathrm{~s}$

4. Pierre SCHAEFFER: TRIÈDE FERTILE: Strette

$1 \mathrm{~m} 21 \mathrm{~s}$

5. Jacques LEJEUNE: PARAGES: Étude de matière, d'espace et de rythme

6. Jean SCHWARZ: ERDA: 54 oiseaux

7. Pierre HENRY: VARIATIONS POUR UNE PORTE ET UN SOUPIR: Chant I

$1 \mathrm{~m} 16 \mathrm{~s}$

$1 \mathrm{~m} 14 \mathrm{~s}$

8. Jean SCHWARZ: ERDA: Variations

9. Pierre HENRY: MOUVEMENT-RYTHME-ÉTUDE: 3e adagio

10. Pierre HENRY: MOUVEMENT-RYTHME-ÉTUDE: Danse

11. Pierre HENRY: MOUVEMENT-RYTHME-ÉTUDE: ler adagio

12. Karlheinz STOCKHAUSEN: TELEMUSIK

$27 \mathrm{~s}$

$1 \mathrm{~m} 24 \mathrm{~s}$

$1 \mathrm{~m} 08 \mathrm{~s}$

$50 \mathrm{~s}$

$1 \mathrm{~m} 30 \mathrm{~s}$

$36 \mathrm{~s}$

13. François BAYLE: GRANDE POLYPHONIE: Fugues doubles

14. Jean SCHWARZ: ERDA: In memoriam

$1 \mathrm{~m} 01 \mathrm{~s}$

$55 \mathrm{~s}$

15. Pierre HENRY: VARIATIONS POUR UNE PORTE ET UN SOUPIR: Étirement

$58 \mathrm{~s}$

16. Bernard PARMEGIANI: DE NATURA SONORUM:

Matières induites

17. Jacques LEJEUNE: PARAGES: Étude de matière, d'espace et de rythme

$1 \mathrm{~m} 30 \mathrm{~s}$

$1 \mathrm{~m} 16 \mathrm{~s}$

18. Bernard PARMEGIANI: DE NATURA SONORUM:

Points contre champs

$2 \mathrm{~m} 30 \mathrm{~s}$

sur des habitudes d'écoute mieux maîtrisées. Les résultats s'en sont trouvés par conséquent enrichis, et le classement des réponses s'est fait de manière plus efficace. Il ne s'agit pas ici d'un biais expérimental en raison de la nature de la tâche demandée aux sujets. Le choix de ces derniers est conséquent avec les objectifs visés.

\section{B) Les extraits}

Dix-huit extraits de musique électroacoustique ont été retenus pour cette expérience (voir figure 1). Ces extraits varient entre 27 secondes et 2 minutes 30 secondes, et proviennent de compositeurs européens ayant des styles variés. 
La durée totale des extraits est d'un peu plus de 20 minutes, pour une durée moyenne par extrait de 1 minute 6 secondes.

Ce sont des extraits et non des œuvres entières qui ont été choisis pour l'expérience. Les raisons de ce choix sont simples. Tout d'abord, le but de l'expérience est de confronter des sujets à une grande diversité de styles, de formes musicales et de matériaux sonores. Cela, afin de recueillir un vaste échantillonnage de réponses à partir desquelles des critères perceptifs pourront être identifiés. Ensuite, comme la consigne était libre, on ne pouvait proposer des œuvres entières au risque d'alourdir considérablement la tâche des sujets. Cela aurait imposé une disponibilité et une charge de travail beaucoup trop exigeantes. Une description détaillée n'est possible qu'à la condition que les extraits ne soient pas trop longs. Enfin, il n'existe pas non plus d'outils méthodologiques appropriés qui conviennent à l'analyse d'œuvres entières dans le cadre d'une expérience. S'en tenir à des extraits est certes un risque, mais un risque nécessaire pour parvenir à des résultats certains. Nous ne pourrons par conséquent que supposer que ces résultats s'appliquent à des œuvres entières, quitte à réaliser une contre-expérience lorsque des outils expérimentaux plus adaptés auront été mis au point.

Deux critères ont tout de même été posés pour le choix des extraits :

- Ils devaient constituer un tout perceptif, c'est-à-dire posséder une unité au même titre que s'il s'agissait d'une œuvre en elle-même. Même sortis de leur contexte, il fallait pouvoir entendre ces extraits comme quelque chose d'autonome.

- Les extraits devaient ensuite s'opposer le plus possible sur le plan formel, afin de refléter de multiples tendances aux niveaux des matériaux, de la construction formelle et de la composition, bref de caractériser la diversité des œuvres électroacoustiques.

Nous croyons que les extraits sélectionnés répondent à ces critères. Ce qui compte, ce n'est pas la diversité des compositeurs des extraits retenus; ce qui compte, c'est la diversité des extraits musicaux eux-mêmes. On pourra objecter que la plupart des compositeurs choisis relèvent d'une esthétique européenne, et que par conséquent les extraits musicaux ne rendent compte que d'une partie du genre. Ce choix s'explique par la relative pauvreté du catalogue des disques consacrés à des œuvres électroacoustiques, surtout justement en dehors de l'Europe. Mais, comme on le verra à la lumière du très grand nombre de réponses obtenues, il est permis de penser qu'une diversification encore plus poussée des extraits n'aurait pas pour autant entraîné des résultats significativement différents. 


\section{C) Le protocole}

La tâche demandée aux sujets était de décrire dans leurs propres mots tout ce qui leur semblait efficace et réussi, et tout ce qui ne l'était pas, dans la série d'extraits musicaux qu'on leur donnait à entendre. Il s'agissait bien de décrire, et non d'associer librement des impressions ou des sentiments. Les sujets devaient être en mesure d'identifier le plus clairement et le plus précisément possible les éléments sonores concernés par leur jugement.

Deux bandes ont été créées, chacune d'elles contenant les mêmes extraits, mais dans un ordre différent afin d'éviter les effets d'ordre. Un bande était remise à chaque participant qui pouvait alors procéder à la réalisation de la tâche de la manière qui lui convenait le mieux. Chaque sujet pouvait ainsi écouter les extraits à son rythme, autant de fois qu'il lui plaisait, et rédiger la description à son gré. L'écoute des extraits se faisait dans des conditions choisies par le sujet et non imposées à celui-ci. De la sorte, sa disponibilité était optimale, l'appréciation des critères qui interviennent dans l'écoute pouvait s'effectuer plus précisément et plus justement.

$$
* * *
$$

\section{LES RÉSULTATS}

\section{A. Description générale}

Le dépouillement des résultats a d'abord consisté à effectuer une analyse de contenu. Cette analyse a pour but de classer la masse des réponses obtenues et principalement d'identifier la nature de chacune de celles-ci. Il s'agit donc de regrouper en grands types généraux les réponses descriptives pour tous les extraits musicaux, et pour tous les sujets.

L'analyse de contenu a fait ressortir les quatre types suivants :

- Critères techniques

- Critères impressifs

- Critères sonores

- Critères formels

Nous allons brièvement décrire chacun de ces critères avant de les analyser plus amplement. 


\section{Critères techniques}

Nous avons regroupé sous l'appellation «critères techniques» toutes les réponses qui s'attardaient à qualifier l'extrait musical en fonction de critères propres à la "fabrication électroacoustique». Il était à prévoir, en effet, que des compositeurs électroacousticiens fassent appel à leur oreille technicienne pour juger l'extrait musical. La qualité de réalisation est ainsi évaluée, la technique de fabrication jugée, le procédé de manipulation sonore qualifié. Sous ce type, nous avons donc regroupé toutes les réponses qui portaient un jugement de valeur sur la réalisation technique proprement dite.

\section{Critères impressifs}

Les «critères impressifs» regroupent toutes les réponses qui décrivent une impression sans la référer à un élément sonore précis. Le sujet qualifie l'extrait musical dans son entier, tente de cerner le sentiment que lui inspire le déroulement sonore. Ces impressions ne rendent pas véritablement compte des critères perceptifs en jeu, principalement dans leur relation avec le trait perceptif, l'élément sonore qui les a induites. Les réponses sont donc le plus souvent floues, vagues, et traduisent un climat ou une ambiance dégagée par l'extrait musical.

\section{Critères sonores}

Les «critères sonores» sont très nombreux. Nous regroupons dans cette catégorie toutes les réponses qui qualifient les éléments sonores dont est constitué l'extrait musical. Ces réponses sont généralement précises et décrivent un point particulier dans l'extrait, par exemple un paramètre sonore. Elles sont évidemment très nombreuses, d'une part parce que les extraits provenaient tous de la musique électroacoustique, avec donc une très grande variété au niveau des objets sonores, et d'autre part, parce que les sujets étaient familiers avec le genre électroacoustique. On constate ainsi que le choix du matériau sonore importe tout autant que sa mise en forme dans l'appréciation générale qui est faite du résultat musical.

\section{Critères formels}

Enfin, dernière catégorie, et la plus importante, les «critères formels» sont les plus instructifs tant sur le plan du nombre de réponses que sur celui de leur intérêt. Les critères formels, en effet, décrivent le déroulement, les proportions, les détails de la structure, les lignes de force, bref des éléments d'analyse qui permettent d'approfondir la compréhension formelle de l'extrait. 
Décrivons maintenant plus en détail ces cinq types en présentant les principales sous-catégories qu'ils recoupent, avec des exemples de réponses. Nous verrons mieux ainsi à quoi se rapporte chacun de ces types.

\section{B. Description détaillée}

\section{Critères techniques}

Quatre sous-catégories ont été définies pour ce premier type. Ce sont :

- Mixage

- Qualité technique

- Appareils de traitement

- Manipulation technique

1.1 Mixage : le mixage est la technique qui permet de superposer des voix, des lignes mélodiques différentes, des objets sonores. Cette technique est très importante en musique électroacoustique puisque c'est elle qui permet de créer des masses sonores denses et des contrepoints complexes.

Les réponses incluses dans cette sous-catégorie citent explicitement la technique du mixage et la qualifient. Les réponses: mixages «bien réussi», "égal», «inégal», «bien réparti », "confus», etc., appartiennent à cette sous-catégorie.

1.2 Qualité technique: plusieurs réponses réfèrent à la qualité proprement technique de l'extrait musical entendu. «Distorsion», "richesse de la prise de son», "pertes de niveaux», "qualité d'enregistrement mauvaise» constituent des exemples des réponses regroupées dans cette sous-catégorie. Chacune d'elles décrit un aspect qui concerne un critère de réalisation technique.

1.3 Appareils de traitement : les sujets étant des compositeurs familiers avec les techniques et les appareils électroacoustiques, on devait s'attendre à ce qu'ils reconnaissent et mentionnent certaines utilisations de ces appareils. Ces réponses ont été regroupées ici. À titre d'exemples, mentionnons les réponses suivantes: "abus de la réverbération», "ajout de l'écho", "variation de vitesse sans caractère», "variation de vitesse intéressante dans la trame», etc.

1.4 Manipulation technique : cette sous-catégorie est semblable à la précédente par le fait que les réponses désignent également une technique de réalisation électroacoustique. La principale différence réside dans le fait qu'il n'y a pas de mention de l'équipement ou du type d'appareil utilisé. «Dispositif technique banal», "procédés trop évidents», «mauvais 
montage», «bons fondus-enchaînés», «technique banale», «surutilisation des fréquences moyennes», "fragmentation agaçante», etc., portent un jugement de valeur sur une technique de manipulation sonore reconnue dans l'extrait musical.

\section{Critères impressifs}

Trois sous-catégories relèvent de ce type :

- Qualité

- Référence

- Qualification thymique

2.1 Qualité : les réponses regroupées dans cette sous-catégorie rendent compte d'impressions générales. Ces impressions indiquent que le sujet a ou n'a pas apprécié tel ou tel aspect dans ce qu'il a entendu, mais sa réponse n'indique pas sur quel fait sonore précis repose son impression. La qualité de l'impression est ainsi traduite par des attributs, sans les relier aux éléments sonores concernés. Ainsi, "trop flou», "pâteux», "chaotique», «confus», «homogène», «complexe», «simpliste», «trop primaire», «trop foisonnant», etc., portent un jugement de valeur sur la qualité de ce qui est entendu, mais sans que l'on sache ce qui, exactement, est «simpliste», «flou» ou «homogène». L'impression est ici générale et qualifie l'extrait dans son ensemble.

2.2 Référence : quelques réponses manifestaient un intérêt, ou le plus souvent un désintérêt, par rapport à l'évocation d'un référent dans l'extrait. Parce que l'extrait musical renvoie à quelque chose de trop connu, un style, une époque, une image, le sujet porte un jugement selon que cette référence lui plaît ou non. Les exemples suivants ont été cités pour nos extraits : «trop électronique», «ça sonne Pop», «trop instrumental», «trop circonscrit à une époque, à une école», etc.

2.3 Qualification thymique: les réponses regroupées ici sont semblables à celles qui sont émises dans la sous-catégorie "qualité». Leur utilisation est la même, c'est-à-dire qu'elles qualifient l'extrait musical dans son ensemble, mais sans décrire précisément dans cet extrait ce qui provoque cette impression. Les réponses se distinguent au niveau de la nature de cette qualification. Dans "qualité», la réponse impressive traduit davantage un jugement de valeur. Dans "qualification thymique», la réponse, cette fois, rend compte d'un certain état d'esprit, d'une certaine humeur, d'une qualification de nature plus affective que rationnelle. Voici quelques exemples : «lassant», "dramatique», "énergique», «lancinant et exaspérant », «climat de mystère très prenant », «sons captivants», etc. 


\section{Critères sonores}

Ce type contient un très grand nombre de réponses regroupées en 7 souscatégories. Les critères sonores permettent de détailler les éléments sonores et musicaux proposés à l'écoute. Ces éléments concernent :

- L'espace

- Le rythme

- Le mouvement

- Les types de sons

- La morphologie

S'ajoutent deux autres sous-catégories :

- La combinaison des éléments

- La qualité sonore

3.1 L'espace : cette sous-catégorie renvoie à la manière dont les éléments se combinent dans l'espace sonore. Lorsque les éléments sonores établissent certains rapports de distance entre eux, avant-arrière, gauche-droite, plans successifs, etc., une impression d'occupation de l'espace se dégage. Plusieurs réponses renvoient ainsi à la qualité de la définition spatiale des extraits musicaux. Pour préciser davantage cette qualité, nous avons subdivisé ces réponses en deux groupes, celles concernant les plans sonores, avec, par exemple, des réponses comme: "plans sonores mal définis", «arrière-plan trop important», "plans sonores bien répartis», etc.; et celles qui décrivent la profondeur: "espace sonore immense», "bonne profondeur», «trop éloigné en profondeur», etc.

3.2 Le rythme : la question du rythme est revenue assez fréquemment dans les réponses descriptives. Soit que la description mentionne une qualité rythmique particulière; soit que le rythme est accolé à un élément sonore quelconque en tant qu'attribut de cet élément. Ainsi, «rythme imprévisible, instable» ou «répétition rythmique trop insistante» rendent compte d'une certaine qualité sur le plan proprement rythmique, tandis que «pédale rythmique irrégulière du son bref» ou "pédale rythmique» précisent la dimension rythmique d'un élément donné.

3.3 Le mouvement : au niveau des éléments sonores, certaines réponses font état des transformations successives lors du déroulement musical. Lorsque ces transformations dessinent des motifs, fluctuent rapidement, s'enchaînent avec beaucoup de variété, une impression de mouvement ou de variation se dégage. Des réponses comme «fourmillement constant plaisant», «beaucoup de mouvement», «beaucoup d'actions bien contrôlées», etc., traduisent une qualité de mouvement dégagé par l'extrait 
musical. Le sujet fait ressortir l'aspect mouvement en tant qu'élément perceptif prédominant dans l'extrait.

3.4 Les types de son: on sait que Pierre Schaeffer (1966) a tenté une classification exhaustive des objets sonores. Il a proposé une grille typologique qui permet, par recoupement de certains critères sonores (durée, masse, facture, continuité ou discontinuité, etc.), d'établir des types sous lesquels pourrait se regrouper la multitude des objets sonores accessibles à l'écoute. Plusieurs des termes (par exemple, «grain», «allure», «trame», «itération», etc.) qu'il a proposés sont passés dans le vocabulaire des musiciens, termes qui sont effectivement utiles pour cerner tel ou tel élément dans un discours musical, pour communiquer un commentaire ou une analyse sur un extrait musical. Plusieurs réponses identifiaient ainsi un critère sonore en recourant explicitement à une dénomination typologique. Ces réponses y gagnent en précision et permettent de bien saisir ce qui était véritablement en jeu lors de la perception du sujet. Des réponses comme: "trame épaisse, bien animée», "sons itératifs électroniques désagréables» ou "pédale pas très uniforme», appartiennent à cette sous-catégorie.

3.5 La morphologie: en décrivant ce qu'ils aimaient ou n'aimaient pas dans l'extrait musical, plusieurs sujets affinaient cette description en recourant à un critère morphologique. Les critères morphologiques sont proches des paramètres sonores habituels : hauteur, durée, intensité et timbre, bien que leur application déborde la définition traditionnelle de ces paramètres. Là encore, ces réponses sont précises et mentionnent un aspect particulier lié à un élément sonore ou au déroulement général. À titre d'exemple: «attaque forte», «trop dense dans le registre moyen», "profils des sons aigus superbes», etc.

3.6 Combinaison des éléments : cette sous-catégorie s'impose à la fois par le nombre de réponses et par leur précision. Elle regroupe toutes les réponses qui traduisent la qualité particulière de la manière dont les éléments sont reliés les uns aux autres dans l'extrait. La combinaison des éléments est décrite selon trois aspects principaux :

- La relation des éléments entre eux : à savoir si les sons sont bien choisis, s'ils établissent des rapports intéressants entre eux : «bon choix des éléments en contrepoint», «manque de rapport entre les sons», etc.

- La transparence: qui indique dans quelle mesure les sons se distinguent les uns des autres, de quelle manière ils se définissent sur la plan de l'écoute les uns par rapport aux autres: «manque de transparence», 
«confusion des voix», "voix de mixage trop imbriquées», «contrepoint très équilibré», etc.

- L'homogénéité : ne critique plus le choix des éléments en tant que tel, mais décrit le résultat sur le plan de l'unité des rapports établis entre les sons. L'homogénéité s'intéresse à la cohérence du résultat sonore: «homogénéité des différents matériaux», «trop disparate», "manque de cohérence entre les matériaux, entre les éléments», etc.

3.7 Qualité sonore: enfin, dernière sous-catégorie des critères sonores, la "qualité sonore» se subdivise elle-même en trois groupes. Chacun décrit un attribut particulier des sons en général entendus dans l'extrait. Globalement parlant, nous avons regroupé dans cette catégorie toutes les réponses qui qualifiaient «les sons», sans plus de précision. Les trois groupes sont:

- La richesse : ce terme sert souvent à désigner l'impression produite par un son ample, épais, avec une belle sonorité. On parle alors de «timbre riche», de "matière sonore très riche», de «sons riches, épais», etc.

- La couleur: ce terme s'applique à la qualité particulière du timbre. La couleur sonore caractérise ce dernier, un peu de la même manière que la couleur sur le plan visuel rend compte de la qualité des objets. Des réponses comme "couleur sonore plaisante», «sons brillants et ronds» ou «son terne» identifient bien ce qu'il faut entendre par couleur sonore. Nous avons également inclu dans ce groupe les réponses qui mentionnaient la texture: «texture granuleuse», "couleur de grain agréable», «texture touffue», etc.

- La sonorité : sous ce groupe, sont réunies toutes les réponses qui décrivent la qualité sonore non plus sur le plan de la richesse ou de la couleur mais sur celui de la sonorité proprement dite. Ces réponses traduisent de diverses manières ce que l'on avait coutume d'appeler le timbre, terme dont la définition fait toujours problème. Pour notre part, les réponses qui utilisent indistinctement les mots «sons», "sonorité » ou «timbre» présentent toutes une parenté de sens suffisante pour justifier qu'on les regroupe ici: «beaux sons», "sonorité sale», «timbre imprécis», etc.

\section{Critères formels}

Dernier grand type de nos critères perceptifs, les critères formels se subdivisent en 8 sous-catégories. Ces sous-catégories rendent compte cette fois des descriptions qui s'attachent aux données formelles exprimées dans l'extrait. 
Les 8 sous-catégories sont:

- Le relief

- L'articulation

- Le début et la fin

- Le développement formel

- Le jeu

- Les changements formels

- La construction

- Les proportions

4.1 Le relief : nous avons regroupé sous le terme «relief» les descriptions qui concernent le procédé formel consistant à mettre en évidence ou à faire émerger un élément particulier par rapport à l'ensemble. Un élément est mis en relief lorsqu'il surgit tout à coup dans le flux sonore, lorsqu'il se découpe sur le fond sonore. Plusieurs réponses mentionnaient le relief comme un critère d'appréciation pertinent, pouvant rendre compte de l'impression ou de l'impact produit lors de l'écoute. Des exemples de ces réponses sont: "relief des éléments percussifs», "émergence d'un motif répétitif», «manque d'impact des figures sonores», etc.

4.2 L'articulation: l'articulation est un critère important. C'est celui qui indique, décrit, la qualité d'enchaînement des changements successifs. L'articulation joue un rôle primordial sur le plan formel; elle détermine le suivi musical et imprègne l'écoute de la qualité propre du déroulement musical. Plusieurs réponses mentionnaient ainsi la qualité des articulations : "bons enchaînements", "figures bien articulées", "transition habile et efficace», «bonne transition d'un son à l'autre», etc.

4.3 Début et fin : une œuvre musicale débute, dure et se termine; à plus forte raison un extrait musical. La manière propre dont débute ou se termine un déroulement musical constitue un critère perceptif pertinent. Le début ou la fin est considéré en tant que tel et joue un rôle important sur l'appréciation de l'extrait. Voici quelques exemples de ces réponses: «bonne conclusion», "cadence finale bien amenée», «début trop brusque», etc.

4.4 Développement: le développement, comme le nom l'indique, renvoie aux réponses qui concernent le déploiement des parties et des éléments dans le temps, la progression formelle de l'extrait musical. Trois groupes de réponses subdivisent cette sous-catégorie :

- La construction du discours : fournit des indications sur l'organisation formelle de l'extrait à un niveau général. La construction tente de résu- 
mer le parcours de l'extrait sur le plan de son déroulement, du discours. Par exemple: «croissance rythmique et dynamique par paliers», «répétitions trop entendues» ou "densité bien contrôlée» permettent de cerner davantage la nature du développement formel soumis à l'écoute.

- La direction : de la même façon, les réponses regroupées ici font ressortir la présence ou l'absence d'une direction contrôlée dans le développement musical. Lorsqu'une ligne directrice est proposée à l'écoute ou lorsqu'au contraire, le développement musical semble ne conduire nulle part, semble être soumis à une construction aléatoire, on constate que cet aspect intervient dans l'appréciation qui est faite de l'extrait. Le critère de la direction, d'une orientation formelle établie par le compositeur, apparaît comme un critère perceptif et d'analyse important. Des réponses comme : «direction bien conduite», «excellente augmentation de la densité pour mener à la fin», "progression qui n'aboutit pas», "ne va nulle part», etc., soulignent la primauté accordée à une articulation soignée des éléments dans le temps, à une construction ordonnée des parties du discours, à un développement sonore qui guide l'écoute.

- La durée : contrairement à la musique instrumentale, où l'importance de la durée se fait sentir en raison des contraintes de la notation, la perception de nos extraits de musique électroacoustique n'a donné lieu qu'à un nombre restreint de réponses sur la durée de ce qui est entendu. Lorsque l'extrait ne plait pas, la durée est quelquefois impliquée, mais le plus souvent ce sont d'autres critères qui expliquent le désintérêt.

Les réponses qui mentionnent explicitement la durée ont été incluses dans la sous-catégorie développement, car elles qualifient le plus souvent l'extrait musical dans son ensemble.

4.5 La jeu: ce terme, «jeu», revient fréquemment pour décrire un mode particulier de combinaison entre des éléments sonores, un recours formel autour duquel la séquence musicale s'organise. Le plus souvent, la mention d'un jeu quelconque entre les éléments est perçue comme un critère d'appréciation positif. Voici quelques exemples de ces réponses: «jeu rythmique sur différents types de sons», «bon jeu des hauteurs», «figures animées, «jeu renouvelé», «dialogue intéressant entre les deux voix», etc.

4.6 Les changements formels : par «changements formels», nous entendons toutes les réponses qui font mention d'une variation ou d'un mouvement formel dans le flux musical. "Variations continuelles sur les mêmes objets», «variations spatiales intéressantes» ou «mouvements désor- 
donnés » fournissent quelques exemples de la manière dont les changements formels sont appréciés dans un extrait musical.

4.7 La construction: les réponses de ce groupe désignent la construction formelle à un niveau général. Elles fournissent, en quelque sorte, une approche du portrait global de l'extrait sur le plan de sa structuration.

Nous avons également utilisé le terme "construction» dans la sous-catégorie «développement», mais c'était en relation avec le discours, c'est-à-dire la nature de la construction des parties opposée à son déroulement dans le temps. Ici, les réponses décrivent la structure, la construction, non plus par rapport à son articulation dans le temps mais de manière statique. La description porte généralement sur un attribut particulier de cette construction comme, par exemple: «bon contraste entre trame et rythme martelé», "rapport fond/ forme mal défini», «ensemble contrapuntique», etc.

4.8 Les proportions : enfin, dernier critère formel, «les proportions» était une sous-catégorie à laquelle il fallait s'attendre. Ce critère est cohérent avec les autres, principalement ceux qui portent sur le développement, la construction et l'articulation. Il désigne la manière dont les parties s'équilibrent entre elles et plusieurs réponses mentionnaient d'ailleurs explicitement le terme «équilibre»: «bon équilibre du rapport fond/forme», «bon équilibre des voix», etc.

La figure 2 présente la récapitulation de ces critères, établis à partir des réponses recueillies lors de l'expérience.

\section{UNE GRILLE D'ANALYSE}

\section{A. Les critères d'analyse}

Les critères perceptifs que nous venons de présenter constituent donc, en quelque sorte, les "points d'intérêt» qu'un auditeur détecte lors d'un acte d'écoute. Ce sont là, d'après les résultats de notre expérience, les appuis sur lesquels repose la perception d'une musique électroacoustique. Dès lors, il est tentant de penser que ces critères, nombreux et généraux, puissent servir de base de comparaison pour analyser une œuvre musicale, qu'elle soit électroacoustique ou non. En l'absence de méthodes d'analyse pouvant englober efficacement les musiques contemporaines dans leur ensemble, peut-être ces critères peuvent-ils servir de point de départ pour comprendre l'organisation et le déroulement de ces musiques. Pourrions-nous donc, à partir de nos critères perceptifs, proposer une grille d'analyse? Une grille d'analyse dont le but serait simplement de recenser les principaux éléments en jeu et de comprendre leurs relations. 
Figure 2

Récapitulation des critères perceptifs

\begin{tabular}{|c|c|}
\hline Critères techniques & $\begin{array}{l}\text { - Mixage } \\
\text { - Qualité technique } \\
\text { - Appareils de traitement } \\
\text { - Manipulation technique }\end{array}$ \\
\hline Critères impressifs & $\begin{array}{l}\text { - Qualité } \\
\text { - Référence } \\
\text { - Qualification thymique }\end{array}$ \\
\hline Critères sonores & 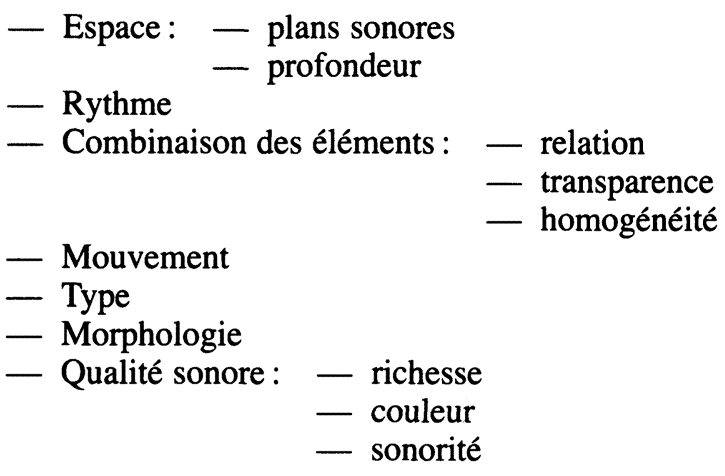 \\
\hline Critères formels & $\begin{array}{l}\text { - Relief } \\
\text { - Articulation } \\
\text { - Début-fin } \\
\text { - Développement: } \\
\\
\\
\text { - } \text { - durée } \\
\text { - construction du discours } \\
\text { - Changements formels } \\
\text { - Construction } \\
\text { - Proportions }\end{array}$ \\
\hline
\end{tabular}

Cependant, une telle grille, si elle est susceptible de guider le travail de compréhension d'une structure musicale, ne peut permettre en revanche d'établir un jugement sur les éléments étudiés, si elle ne repose que sur nos seuls critères perceptifs. Identifier ces éléments dans une structure musicale est une chose; pouvoir analyser leur rôle et leur efficacité en est une autre. Le recense- 
ment seul des éléments est insuffisant pour saisir le sens même d'une organisation musicale et surtout, pour permettre la comparaison de diverses œuvres musicales, ce qui, en définitive, est le véritable but d'un travail d'analyse.

Pour construire une grille d'analyse à partir de nos critères perceptifs, il faut donc proposer également d'autres critères, que nous appellerons cette fois des critères d'analyse. Pour définir cette deuxième série de critères, nous avons procédé en recherchant comment s'opposent les critères perceptifs. En effet, la lecture d'une réponse comme «bon équilibre des voix» ou encore "sonorités désagréables» indique bien dans quelle direction l'auditeur a fait porter son écoute. Mais cela ne nous indique pas, en revanche, pourquoi l'équilibre des voix était bon ou pourquoi les sonorités étaient désagréables. Or, c'est là justement ce qu'il nous importe de savoir pour être en mesure de porter un jugement sur l'œuvre entendue. Par contre, si on oppose les réponses «discours trop décousu» et "bonne continuité du discours», ou encore les réponses «sons trop disparates» et «les sons vont bien ensemble», on constate facilement que le facteur de jugement ici porte sur un critère d'unité. On comprend mieux alors, dans cet exemple, que ce qui permet de dire que le discours est bien suivi ou trop décousu est fonction d'un jugement sur l'unité de ce discours, l'unité étant ici un critère d'analyse. Ce sont ces directions qu'il nous faut à présent rechercher, afin d'échapper à la nature subjective des réponses et de construire une grille d'analyse efficace.

La méthode a donc consisté à opposer entre eux tous les critères perceptifs, selon qu'ils portaient un jugement négatif ou positif. L'analyse de ces oppositions a ensuite permis d'identifier trois critères principaux. Ces critères d'analyse sont : l'unité, la variété et l'écoute.

\section{Critère d'unité}

Lors d'une écoute, des liens sont implicitement créés par l'auditeur entre les éléments sonores entendus. Lorsque ces liens n'apparaissent pas clairement, lorsque les relations entre les éléments semblent soumises à la seule loi du hasard ou bien ne semblent pas maîtrisées, l'écoute s'égare, n'est plus orientée et l'impression d'ensemble en souffre. L'unité traduit le besoin d'une certaine hiérarchie dans l'information qui est offerte à l'auditeur, le besoin d'un certain ordre dans la conduite du déroulement.

Le critère d'unité se subdivise en trois sous-catégories :

- Manque : lorsque l'extrait musical est confus, désordonné, que les éléments sont enchevêtrés, il y a un manque d'unité sur le plan perceptif. 
C'est ce que traduisent des réponses comme : «fouillis», «trop hétérogène », «trop disparate», « décousu», etc.

- Excès: à l'inverse, lorsque l'extrait est trop uniforme, qu'il n'offre pas suffisamment de nouveauté à l'écoute, il y a un excès d'unité. «Trop pareil», «choix d'éléments sonores trop semblables», etc., laissent comprendre que l'extrait ne présente pas d'intérêt en raison de la trop grande uniformité du déroulement sonore.

- Équilibre : enfin, entre le manque d'unité et l'excès d'unité, il y a place pour un juste équilibre que soulignent des réponses comme : «bon rapport entre les éléments», «homogène», etc.

\section{Critère de variété}

S'il y a un rapport entre les éléments à considérer durant une analyse musicale, il y a aussi la manière dont ils évoluent dans le temps qu'il importe de définir. C'est le critère de variété qui va permettre de saisir et d'évaluer les modalités du parcours musical, l'enchaînement et le renouvellement des séquences et des objets. Le critère de variété s'intéresse donc à la qualité des transformations qui sont proposées à l'écoute. Le critère de variété se divise selon les mêmes sous-catégories que le critère d'unité :

- Manque : cette sous-catégorie rend compte de la quantité d'information présente dans le déroulement musical et du renouvellement de cette information. Lorsque le flux musical demeure trop semblable à lui-même, lorsqu'il ne propose pas suffisamment d'éléments susceptibles de relancer l'intérêt musical, il n'y a pas assez de variété, de changements. Le manque de variété, et le manque d'intérêt qui s'ensuit, s'expriment par des réponses comme: «information pas assez renouvelée», "pas assez de changements de timbres», "pas assez diversifié».

- Excès : à l'opposé, la situation est celle où, cette fois, trop d'informations surchargent l'écoute, la rendent confuse, là où la trop grande variété des éléments et des enchaînements rend difficile la compréhension du déroulement musical. "Mouvements désordonnés», «agité», "rythme emporté» soulignent la difficulté à percevoir le renouvellement, le passage trop rapide de ce qui est donné à entendre.

- Équilibre : entre une redondance trop marquée et un renouvellement trop imprévisible, un juste équilibre dans la variété offerte à l'écoute se traduit par des réponses comme : "prévisible tout en étant varié», "varie beaucoup avec peu de moyens», «beaucoup d'actions bien contrôlées», etc. 


\section{Critère d'écoute}

Les critères d'écoute sont ceux décrivent comment l'extrait a plu ou déplu par rapport à l'impact produit sur l'écoute, par rapport à la manière dont l'oreille est dirigée et stimulée. Ils fournissent plusieurs indications précieuses concernant le déroulement temporel de l'extrait sonore.

Les réponses qui traduisent les critères d'écoute sont regroupées dans trois sous-catégories :

a) Densité : la quantité d'information soumise à l'écoute crée une densité qui peut être qualifiée pour elle-même. Lorsqu'une réponse mentionnait l'impact sur l'écoute d'un critère de densité, nous l'avons incluse dans cette sous-catégorie. "Trop grande quantité d'informations», "fouillis», "pêle-mêle», "bonne densité», etc., indiquent le confort ou l'inconfort de l'oreille par rapport à la quantité d'informations présentes dans l'extrait. La densité rend compte de la quantité d'éléments en jeu à un instant donné. Il s'agit d'un critère important sur le plan de l'écoute. La qualité de cette dernière, en effet, dépendra en grande partie de la capacité de l'auditeur à assimiler l'information d'ensemble qu'il entend. Puisqu'il s'agit d'une quantité d'éléments, nous utiliserons les mêmes sous-catégories que les critères «unité" et "variété» pour détailler la densité :

- Excès : lorsque la densité sonore est trop importante, la quantité d'information surcharge la perception et nuit à son intelligibilité. La qualité de l'écoute en souffre, ce que traduisent des réponses comme: "confus», «complexe», «qualité sonore trop dense», «confusion des voix», «surcharge», etc.

- Manque : la situation inverse laisse trop peu de «substance» à l'oreille. Une densité d'éléments peu importante lasse l'écoute en raison du peu d'intérêt qualitatif du déroulement sonore proposé. "Simpliste», "sons trop minces», etc., sont quelques exemples des réponses qui mentionnent une densité trop faible.

- Équilibre: lorsque la quantité d'éléments proposés à l'écoute est suffisamment bien répartie dans le temps pour que le parcours soit correctement saisi, on peut parler d'équilibre de la densité. Un tel équilibre assure, d'après les réponses descriptives, une qualité d'écoute agréable : densité bien contrôlée», "masse très pleine», «bonne répartition de la densité», «bonne répartition des moments denses et aérés», etc. 
b) Intérêt : plusieurs réponses se rapportaient à l'intérêt provoqué par l'extrait musical lors de l'écoute. Ces réponses traduisent la stimulation de l'écoute de manière générale: "l'intérêt se réveille», "force l'écoute attentive», «banalité de l'information", "relance de l'intérêt», "sans intérêt», «trop redondant», etc. L'appréciation d'une séquence musicale s'exprime très souvent par l'intérêt suscité chez l'auditeur. L'attention de ce dernier est maintenue, des éléments ressortent pour soutenir la motivation. L'intérêt engendré se divise en deux catégories, selon la manière dont il se manifeste : intérêt soutenu (et, par opposition, non soutenu) et intérêt relancé (par opposition, non relancé).

- Soutenu : lorsque l'intérêt est constamment sollicité, l'attention se maintient et l'auditeur demeure concentré sur ce qu'il entend. Plusieurs facteurs peuvent favoriser ce maintien de l'intérêt, comme en témoignent les réponses suivantes: «sons captivants», «fourmillement constant plaisant», «dialogue intéressant entre les deux voix», etc.

- Non soutenu : à l'inverse, l'intérêt peut aussi ne pas se maintenir, l'attention se relâche, la concentration se perd, le plaisir de l'écoute s'atténue. Là encore, plusieurs facteurs peuvent concourir à expliquer le désintérêt vis-à-vis l'extrait musical: «forme trop stable», «trop linéaire», «trop primaire», etc.

- Relancé : certains procédés formels peuvent orienter l'écoute et provoquer celle-ci en introduisant des contrastes, des directions nouvelles, des relations différentes. L'intérêt est ainsi soutenu mais de manière plus ponctuelle, en fonction du déroulement et de l'organisation générale de l'extrait musical. "Jeu de contraste intéressant», «jeu renouvelé», «jeu inventif», etc., démontrent que les modalités particulières du jeu des éléments sonores, leurs modes d'organisation, sont particulièrement importants pour une relance constante de l'intérêt musical.

- Non relancé : au contraire, les procédés formels auxquels recourt le compositeur peuvent s'avérer inefficaces pour relancer l'intérêt de l'auditeur. Certains motifs de cet échec transparaissent à travers les réponses suivantes: «manque d'impact des figures sonores», "répétitions trop attendues", "procédés trop évidents», "pas assez diversifié», etc.

c) Conduite de l'écoute: les sujets ont, à quelques reprises, insisté sur la conduite de l'écoute, sur la manière dont le déroulement musical dirige l'écoute. Ces réponses sont intéressantes car elles fournissent un critère d'analyse qui qualifie le discours général de l'extrait, la manière dont les événements sonores s'enchaînent les uns aux autres dans le temps, et leur 
impact sur l'écoute. Des exemples de réponses sont: «l'information n'est pas suffisamment renouvelée», "surprise au début», «bonne polarisation de l'écoute», "manque d'un point culminant», "pas assez de changements de timbre», «on se sent guidé, le compositeur sait où il va», "manque de contexte», "n'importe où, n'importe comment», etc. La conduite traduit la manière dont l'oreille est dirigée, entraînée, canalisée dans le flux musical grâce aux caractéristiques formelles du déroulement de l'extrait. La conduite de l'écoute se traduit dans les réponses par les descriptions qui désignent un état «avant» et «après», c'est-à-dire comment s'enchaînent les parties et les éléments, et le degré de cohérence entre ces états successifs.

La cohérence du déroulement influence le sentiment de direction musicale, l'impression positive que le discours évolue de manière conséquente, la conviction que le compositeur maîtrise le parcours temporel de sa musique. «Bonne distribution des éléments dans la durée», «bonne transition entre les trames», "cadence finale bien amenée», «déroulement bien conduit», «succession des séquences bien menée», etc., renvoient à cette fonction perceptive qui recherche la continuité musicale.

Par opposition, une écoute non conduite provoque des réponses catégoriques : «manque de direction», "n'évolue pas vers quelque chose», «discontinuité du matériau», «éparpillement des éléments», «progression qui n'aboutit pas», «ne va nulle part», etc.

Ce troisième critère d'analyse, l'écoute, est quelque peu particulier puisqu'il regroupe des réponses qui concernent l'écoute en elle-même, tout en constituant un critère sur lequel s'opposent des réponses appartenant aux critères sonores et formels.

La figure 3 présente la récapitulation des critères d'analyse.

\section{B. Analyse globale et analyse détaillée}

Nous possédons à présent deux entrées d'une grille d'analyse. D'une part, des critères perceptifs (principalement, les critères sonores et formels, les critères techniques étant trop spécifiques au genre électroacoustique, et les critères impressifs trop dépendants de l'auditeur) qui se recoupent avec trois critères d'analyse, l'unité, la variété et l'écoute. Toutefois, il nous reste une dernière modalité d'organisation à considérer avant de construire en bonne et due forme une telle grille d'analyse, c'est le niveau d'analyse auquel s'appliquent les critères. En effet, certains critères demeurent à un niveau descriptif général, global, et considèrent l'extrait musical dans son entier. Par exemple, «dis- 


\section{Figure 3}

Récapitulation des critères d'analyse

\begin{tabular}{ll}
\hline - Unité : & - excès \\
& - manque \\
& - équilibre \\
- Variété : & - excès \\
& - manque \\
& - équilibre \\
- Écoute : & - densité : $\quad$ - excès \\
& $\quad$ manque \\
& - équilibre \\
& - conduite, non conduitenu, non soutenu \\
\hline
\end{tabular}

cours bien mené» ou «jeu rythmique imprévisible» sont des réponses qui relèvent d'une considération d'ensemble. Par contre, «bonne transition entre les trames» et «cadence finale bien amenée» mentionnent plus précisément les éléments considérés dans le jugement. Ils relèvent cette fois d'une analyse plus détaillée.

Il nous faut donc à présent définir les niveaux d'analyse globale et détaillée en regroupant les critères perceptifs qui s'appliquent à un ou l'autre de ces niveaux.

\section{Analyse globale}

L'analyse globale est celle qui qualifie l'extrait musical dans son ensemble. Elle porte un jugement sur la totalité de l'extrait sans entrer dans le détail des éléments qui concourent à forger ce jugement.

Voici, parmi nos principaux critères perceptifs (sonores et formels), ceux qui se prêtent à une analyse globale :

1.1 Critères sonores : plusieurs sous-catégories ont été définies pour les critères sonores. Parmi celles-ci, les suivantes relèvent de l'analyse globale :

- Espace : les modalités spatiales dégagées par l'extrait musical sont décrites à un niveau global. Des descriptions comme : «espace sonore immense», «plans sonores bien répartis», «bonne profondeur», etc., 
qualifient de manière générale le paramètre de l'espace dans l'extrait musical, sans plus préciser sur quoi repose la dimension spatiale perçue.

- Rythme : plusieurs réponses décrivaient la qualité rythmique générale de l'extrait: "rythmique inégale et hésitante», "rythme emporté», "rythme trop redondant», etc., fournissent quelques exemples de ces réponses. On doit noter cependant que certaines réponses renvoient à un niveau plus précis. Par exemple, "pédale rythmique» ou "motifs rythmiques agréables » associent la qualité du rythme à des éléments sonores donnés. Malgré cela (et en plus de la relative rareté de ces réponses concernant le rythme), le niveau de description demeure à un degré trop général pour associer le rythme à un facteur d'analyse détaillée.

- Combinaison des éléments : cette sous-catégorie peut renvoyer tout aussi bien à un niveau global qu'à un niveau détaillé. Elle concerne la qualification des éléments constitutifs de l'extrait musical et, à ce titre, la description peut rendre compte de ces éléments aussi bien de manière générale que de manière précise. À un niveau général, nous retrouvons des réponses comme: «bon rapport entre les éléments», "voix de mixage trop imbriquées», "manque de cohérence entre les différents éléments», etc.

- Mouvement : certaines réponses font explicitement mention de la qualité du mouvement impliqué dans l'extrait. Ces réponses se situent à un niveau général et tentent de faire ressortir les différents contours dessinés par les éléments sonores. "Beaucoup d'actions bien contrôlées», "fourmillement constant plaisant", "redondances inutiles", etc., fournissent quelques exemples de ces réponses générales.

- Qualité sonore : on pourrait ici croire que la qualité sonore renvoie à un niveau de description précis. Ce n'est pas le cas. La qualité sonore, d'après notre définition de ce critère, est une qualification générale sur la richesse, la couleur ou la sonorité de l'extrait musical. Lorsque les réponses mentionnent ces paramètres à propos d'un élément sonore précis, nous les regroupons dans la catégorie «typo-morphologie». Des exemples d'analyse globale sur le plan de la qualité sonore sont: "matière sonore très riche», «couleurs sonores uniformes», «sons peu attrayants», etc.

1.2 Critères formels : les critères formels s'attardent sur les modalités d'organisation proprement dites et non plus sur les éléments qui composent l'extrait. Les principaux caractères de la structure formelle sont décrits. Parmi les sous-catégories des critères formels, celles qui se situent à un niveau d'analyse globale sont les suivantes: 
- Relief : les réponses concernant le relief renvoient à un procédé formel qui consiste à mettre en évidence certaines particularités du parcours musical. Ainsi, "émergence d'un motif répétitif», «relief des éléments percussifs», "manque de contraste», etc., mentionnent la manière dont certaines mises en valeur sont réalisées, à un niveau général, dans l'extrait.

- Articulation : la qualité de l'enchaînement des différents traits perceptifs d'une séquence musicale ou sonore se traduit par leur degré d'articulation. Cette sous-catégorie peut tout aussi bien s'appliquer au passage précis d'un élément ou d'une partie vers une autre qu'à la qualité d'ensemble des liens établis entre les états successifs du déroulement sonore ou musical. C'est pourquoi, nous retrouverons l'articulation tant au niveau de l'analyse globale qu'à celui de l'analyse détaillée. Au niveau global, des exemples de réponses sont: «bons enchaînements", "articulation soignée», «bonne transition d'un son à l'autre», etc.

- Développement : les principales réponses formelles au niveau de l'analyse générale se retrouveront sous la catégorie «développement». C'est ici, en effet, que les modalités du développement dynamique de la structure formelle sont décrites. Ces modalités portent sur la durée, la construction du discours et la direction du développement. Voici quelques exemples de réponses: "durée adéquate», «bonne répartition des moments denses et aérés", "séquences rythmiques enchaînées", «évolution du discours bien menée», «bon développement polyphonique», etc.

- Changements formels : les diverses modifications apportées au parcours musical sont souvent relevées dans les réponses. Lorsque ces modifications concernent l'organisation générale, nous les incluons dans la sous-catégorie «changements formels»; lorsqu'elles se rapportent à des éléments sonores précis, elles se retrouvent dans la sous-catégorie «mouvement» des critères sonores. Des exemples de réponses décrivant les changements formels sont: «métamorphose lente», «mouvements désordonnés», «variations spatiales intéressantes», etc.

- Construction : cette catégorie décrit l'organisation générale de l'extrait au niveau de sa construction formelle. La description ne porte plus sur les aspects dynamiques de la construction (comme dans «construction du discours" de la sous-catégorie "développement»), mais plutôt sur les aspects statiques et ce, à un niveau global: «bien construit, vivant», «rapport fond/forme mal défini», «bon rendu de la permanence/variation», etc. 
- Proportions : l'équilibre des différents rapports formels établis dans l'extrait musical est qualifié dans cette sous-catégorie. Ici encore, l'analyse demeure à un niveau global: «bon équilibre du rapport fond/forme», «bon équilibre des voix», «bon équilibre entre les types d'objet», etc.

\section{Analyse détaillée}

Au niveau de l'analyse détaillée, cette fois, les descriptions porteront sur des éléments plus précis, plus spécifiques, dégagés dans le cours du déroulement musical. Les réponses feront donc explicitement mention d'un élément en particulier.

\subsection{Critères sonores :}

- Combinaison des éléments : comme nous l'avions déjà mentionné, la combinaison des éléments peut se rapporter à des détails précis au niveau de la relation, de la transparence ou de l'homogénéité. Voici quelques exemples de ces réponses: "trame trop importante et présente», «manque de définition des notes ", etc.

- Typo-morphologie : nous regroupons ici deux sous-catégories que nous avions présentées séparément dans l'analyse de contenu des résultats de l'expérience. Il existe une certaine parenté de sens entre ces deux termes, la typologie désignant la catégorie où l'on situe les objets sonores afin de les distinguer les uns des autres, la morphologie précisant davantage les caractéristiques sonores qui permettent d'identifier les objets sonores.

Des exemples de description typo-morphologique sont: "trame épaisse», «impulsions mal définies», "trop dense dans le registre moyen», "profils bien définis", etc.

2.2 Critères formels :

- Début-fin: le début et la fin d'une séquence musicale sont souvent qualifiés dans les descriptions. Ils concernent un aspect formel particulier : «fin abrupte», «cadence finale bien amenée», «fin amusante», etc.

- Articulation: lorsque la description désigne une articulation, un enchaînement particulier entre des éléments sonores, elle se retrouve à un niveau d'analyse détaillée. Voici quelques exemples de ces réponses : "bonne transition entre les trames», «figures bien articulées», «apparition d'une résonance bien amenée», etc.

- Jeu: le jeu décrit le fonctionnement des éléments sonores les uns par 
rapport aux autres, leurs modalités de mouvement et de parcours, leur facilité à se mouvoir dans le temps. Cette description précise le plus souvent la nature des éléments concernés: "dialogue entre graves et aigus», «bon jeu des hauteurs», «jeu rythmique sur différents types de son", etc.

Cela complète notre définition des différents niveaux d'analyse, global et détaillé. Nous pouvons donc à présent dresser notre grille d'analyse en opposant les critères perceptifs (en ordonnée) aux critères d'analyse (en abscisse). La grille se divise en deux parties: analyse globale et analyse détaillée (voir les figures 4 et 5 ).

* * *

\section{REMARQUES ET CONCLUSION}

La construction de la grille d'analyse fait donc intervenir deux ordres de description. D'abord, des critères perceptifs qui rendent compte de l'écoute de l'auditeur, des traits perceptifs qu'il retient dans le déroulement sonore proposé. Ensuite, des critères d'analyse qui approfondissent les critères perceptifs, en précisant à la fois leur sens et leur relation à l'extrait musical et aux éléments sonores. La combinaison de ces critères se fait par recoupement. Le résultat de ce recoupement dessine sur la grille d'analyse des cases.

Il va de soi que certains critères perceptifs s'appliquent mieux à certains critères d'analyse qu'à d'autres. Par exemple, le critère perceptif du «mouvement» concerne davantage le critère d'analyse de la variété plutôt que celui de la densité, même si un tel recoupement n'est pas exclu. En ce sens, la grille d'analyse offre beaucoup de souplesse. Dans un premier temps, à l'écoute d'une musique, il s'agit de cerner le niveau considéré, soit global ou détaillé. Par la suite, pour chacun de ces niveaux, il s'agit de déterminer les critères perceptifs qui s'appliquent à l'extrait. Une fois ces critères définis, il reste à les juger à la lumière des critères d'analyse. Ces derniers permettent de dépasser le premier niveau de la description induite par les critères perceptifs et d'en approfondir le sens.

Tous les critères ne vont pas forcément s'avérer pertinents lors d'un travail d'analyse. Cela dépend bien sûr de l'organisation musicale considérée. L'intérêt de la grille d'analyse, c'est que grâce à son caractère général, elle permet une revue systématique des éléments à considérer dans le travail d'analyse; elle permet aussi de cerner de manière rigoureuse sur quoi repose le jugement 


\begin{tabular}{|c|c|c|c|c|c|c|c|c|c|}
\hline \multirow{2}{*}{$\begin{array}{l}\text { Critères } \\
\text { perceptifs }\end{array}$} & \multicolumn{3}{|c|}{ UNITÉ } & \multicolumn{3}{|c|}{ VARIATION } & \multicolumn{3}{|c|}{ ÉCOUTE } \\
\hline & Excès & Équillibre & Manque & Excès & Équillibre & Manque & Densité & Intérêt & Conduite \\
\hline \multicolumn{10}{|l|}{$\begin{array}{l}\text { A) Critères sonores } \\
\text { - Mouvement }\end{array}$} \\
\hline - Rythme & & & & & & & & & \\
\hline - Qualité sonore & & & & & & & & & \\
\hline - Espace & & & & & & & & & \\
\hline - Combinalson des éléments & & & & & & & & & \\
\hline $\begin{array}{l}\text { B) Critères formels } \\
\text { - Proportions }\end{array}$ & & & & & & & & & \\
\hline - Construction & & & & & & & & & \\
\hline - Développement & & & & & & & & & \\
\hline - Articulation & & & & & & & & & \\
\hline - Rellef & & & & & & & & & \\
\hline - Changements formels & & & & & & & & & \\
\hline
\end{tabular}

\section{Figure 4}

Analyse globale 


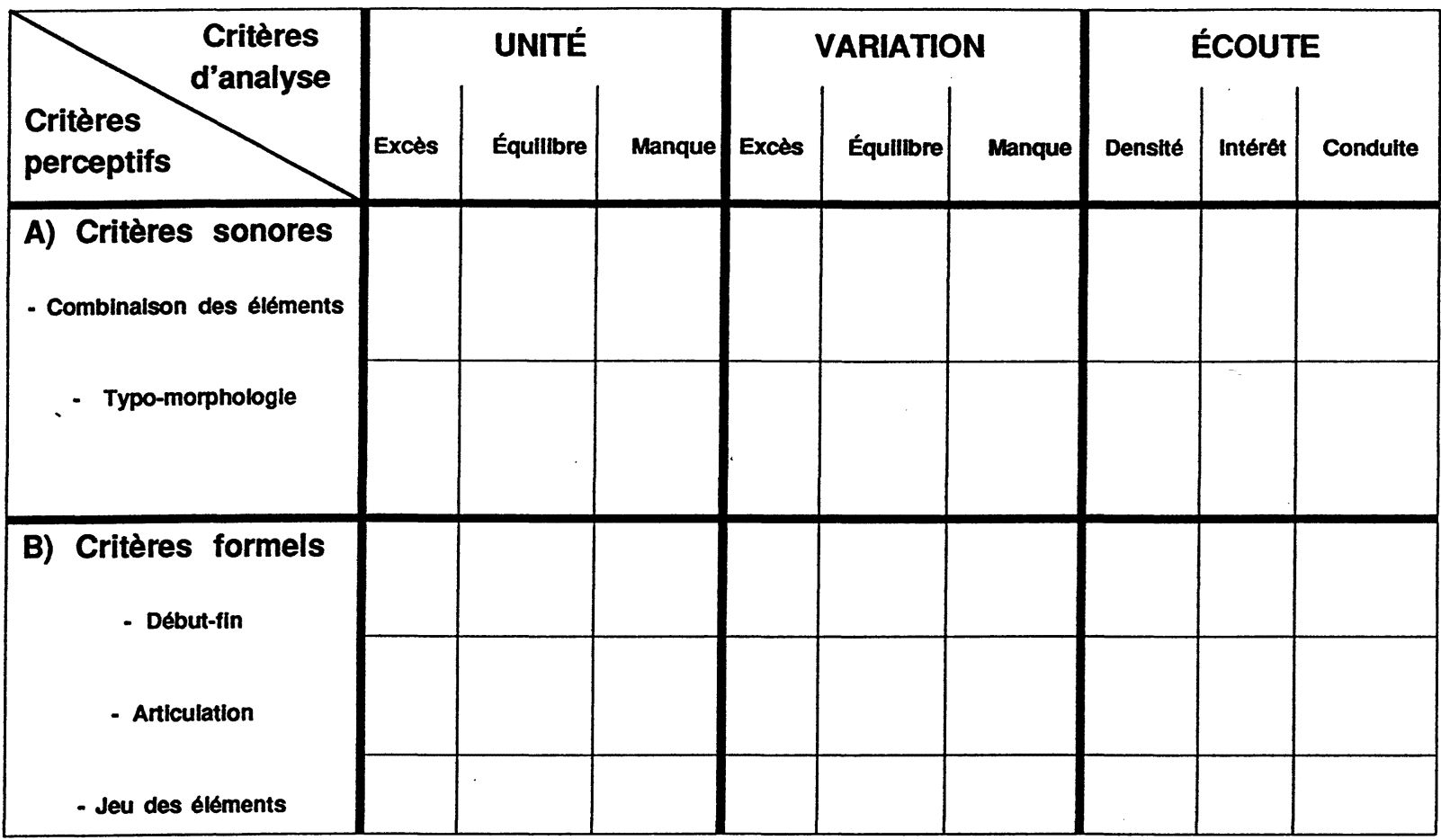

Figure 5

Analyse détaillée 
à porter sur la musique analysée. Avec une telle grille sous les yeux, il est facile de décrire les différents aspects d'une musique, aspects susceptibles de favoriser la compréhension de cette musique. Tout ce qui peut intervenir dans l'analyse, tout ce qui peut permettre d'approfondir la compréhension des facteurs en jeu, est rappelé dans la grille, du moins par rapport aux résultats de notre expérience.

Il faut ensuite remarquer que cette grille tente de s'appliquer à une généralité de situations. Toute structure sonore ou musicale doit pouvoir être analysée à l'aide de ce canevas procuré par la grille. Un tel parti est-il permis en considérant que les critères définis reposent sur des extraits de musique électroacoustique? Si l'intention de la grille est générale, rien ne permet d'affirmer sans précaution qu'elle puisse s'appliquer à toutes les organisations musicales ou sonores. D'autres expériences avec d'autres genres musicaux, des œuvres entières, voire des paysages sonores, pourraient faire surgir d'autres critères et remettre en question l'organisation de cette première grille.

Par contre, les catégories d'analyse indiquées sur la grille ne constituent pas des unités tranchées, rigides, étanches. Les critères laissent un jeu pour l'interprétation, permise en raison du nombre restreint de critères dans la grille. Aussi, chacun pourra appliquer la grille selon la signification qu'il accorde à tel ou tel critère; l'écart ne sera jamais trop grand d'une analyse à l'autre. Ce qui compte principalement, c'est la cohérence de l'analyse pour qu'elle puisse former un tout signifiant. L'utilité et l'objectif d'une telle grille d'analyse, en définitive, serait de permettre d'unifier les critères de comparaison entre diverses analyses et diverses pièces musicales. Le choix des critères d'analyse ne repose plus sur les compétences, les connaissances ou les aptitudes de la personne qui se livre à une analyse musicale. La grille propose un consensus sur les termes et sur la manière de conduire une analyse. Les critères d'analyse forcent une plus grande précision des descriptions, conduisent à approfondir le jugement grâce à la revue systématique des facteurs qui interviennent dans le donné sonore.

Les critères d'analyse permettent enfin d'échapper à une certaine subjectivité des réponses, à les rendre plus explicites. Ainsi, pour comprendre pourquoi un «jeu de contraste» est «intéressant», on peut se demander si c'est à cause de la qualité de la variation, de son effet sur l'écoute, de son unité, etc.

Bien sûr, cette grille n'est qu'un cadre de travail. Sa pertinence ne pourra être vérifiée qu'à travers son utilisation, qu'à travers sa confrontation à des organisations sonores diversifiées. Quoi qu'il en soit, elle s'inscrit dans la tendance de plus en plus pressante de définir des outils d'investigation qui puissent prendre en compte la richesse et la complexité des mondes sonores qui sont aujourd'hui proposés à l'écoute. 


\section{RÉFÉRENCES}

AROM, S.

1985 : «De l'écoute des musiques centrafricaines», Analyse musicale, $1: 35-$ 39.

BREGMAN, A. S.

1978 : "Auditory Streaming : Competition between Alternative Organizations", Perception and Psychophysics, 23, no. $5: 391-398$.

COGAN, R. et ESCOTT, P.

1976: Sonic Design: The Nature of Sound and Music. Englewood Cliffs : Prentice-Hall.

COOPER, G. W. et MEYER, L. B.

1960 : The Rhythmic Structure of Music. Chicago : The University of Chicago Press.

DELALANDE, $\mathrm{F}$.

1976 : «Pertinence et analyse perceptive», dans «Le Traité des objets musicaux 10 ans après », Cahiers recherche/musique, 2: 73-90.

DEUTSCH, D.

1982: The Psychology of Music. New York: Academic Press.

ERICKSON, R.

1975 : Sound Structure in Music. The University of California Press.

LERDHAL, F. et JACKENDOFF, R.

1983 : A Generative Theory of Tonal Music. Cambridge : MIT Press.

PLOMB, R.

1976 : Aspects of Tone Sensation. New York : Academic Press.

SCHAEFFER, $P$.

1966 : Traité des objets musicaux. Paris : Seuil.

THORESEN, L.

1985 : «Un modèle d'analyse auditive», Analyse musicale, 1: 44-60.

WESSEL, D.

1979 : "Timbre Space as a Musical Control Structure", Computer Music Journal, 3 , no $2: 45-52$. 